\title{
Gastric outlet obstruction due to adenocarcinoma in a patient with Ataxia-Telangiectasia syndrome: a case report and review of the literature
}

\author{
Iyore A Otabor ${ }^{1}$, Shahab F Abdessalam², Steven H Erdman ${ }^{3}$, Sue Hammond ${ }^{4}$ \\ and Gail E Besner*1
}

\begin{abstract}
Address: ${ }^{1}$ Department of Pediatric Surgery, Nationwide Children's Hospital and The Ohio State University College of Medicine, Columbus, OH 43205, USA, ${ }^{2}$ Department of Pediatric Surgery, Children's Hospital of Omaha, Omaha, NE 68114, USA, ${ }^{3}$ Division of Gastroenterology, Hepatology and Nutrition, Nationwide Children's Hospital and The Ohio State University College of Medicine, Columbus, OH 43205, USA and ${ }^{4}$ Department of Pathology and Laboratory Medicine, Nationwide Children's Hospital and The Ohio State University College of Medicine, Columbus, OH 43205, USA

Email: Iyore A Otabor - iyore.otabor@nationwidechildrens.org; Shahab F Abdessalam - sabdessalam@chsomaha.org; Steven H Erdman - steve.erdman@nationwidechildrens.org; Sue Hammond - sue.hammond@nationwidechildrens.org;

Gail E Besner* - gail.besner@nationwidechildrens.org

* Corresponding author
\end{abstract}

Published: 12 March 2009

World Journal of Surgical Oncology 2009, 7:29 doi:10.1186/1477-7819-7-29

This article is available from: http://www.wjso.com/content/7/1/29

(C) 2009 Otabor et al; licensee BioMed Central Ltd.

This is an Open Access article distributed under the terms of the Creative Commons Attribution License (http://creativecommons.org/licenses/by/2.0), which permits unrestricted use, distribution, and reproduction in any medium, provided the original work is properly cited.

\begin{abstract}
Background: Ataxia-Telangiectasia syndrome is characterized by progressive cerebellar dysfunction, conjuctival and cutaneous telangiectasias, severe immune deficiencies, premature aging and predisposition to cancer. Clinical and radiographic evaluation for malignancy in ataxiatelangiectasia patients is usually atypical, leading to delays in diagnosis.

Case presentation: We report the case of a 20 year old ataxia-telangiectasia patient with gastric adenocarcinoma that presented as complete gastric outlet obstruction.

Conclusion: A literature search of adenocarcinoma associated with ataxia-telangiectasia revealed 6 cases. All patients presented with non-specific gastrointestinal complaints suggestive of ulcer disease. Although there was no correlation between immunoglobulin levels and development of gastric adenocarcinoma, the presence of chronic gastritis and intestinal metaplasia seem to lead to the development of gastric adenocarcinoma. One should consider adenocarcinoma in any patient with ataxia-telangiectasia who presents with non-specific gastrointestinal complaints, since this can lead to earlier diagnosis.
\end{abstract}

\section{Background}

Ataxia-Telangiectasia (A-T) was first reported in 1926 in three patients that presented with progressive choreoathetosis and ocular telangiectasias [1]. By 1958, it was recognized as a distinct disease process and named ataxiatelangiectasia [2]. The most common initial abnormalities noted are balance and gait problems [3]. Unsteadiness and truncal ataxia typically appear before 3 years of age, with slurred speech by 5 years of age. Patients are usually wheel-chair bound by 10 years of age due to excessive falling coupled with slow reflexes that cause serious bodily injuries. About a third of the patients present with severe immunodeficiencies accompanied by severe sino-pulmonary infections with non-opportunistic organisms, a third 
have moderate immunodeficiencies, and a third have no signs of immunodeficiency [4]. Serum levels of alpha fetoprotein (AFP) are usually increased in A-T patients [4-6]. There appears to be a direct correlation between serum AFP levels and age [7].

While there have been significant improvements in diagnostic modalities for A-T, there is still no cure and treatment is mainly symptomatic. Patients with A-T have a 70250 fold increased risk for developing a lymphoreticular malignancy $[8,9]$. However, clinical and radiographic diagnosis of malignancy in these patients can be difficult because the presentation is usually atypical and may be confused with the infectious processes commonly associated with immunodeficiency syndromes. Six cases of gastric cancer associated with A-T have been reported in the literature in patients ages 14-26 years; of these, four cases were reported in the English literature with the last case reported in 1979 [10]. We present the case of a 20 year old female with ataxia-telangiectasia who was transferred to our institution with a diagnosis of pancreatic abnormality. Further diagnostic evaluation revealed gastric adenocarcinoma that was resected.

\section{Case presentation}

The patient is a 20 year old female that was diagnosed with ataxia-telangiectasia syndrome at 3 years of age. She had severe ataxia and was wheelchair bound by 8 years of age. She presented with a 4 week history of non-bilious emesis, early satiety, decreased appetite and a $35 \mathrm{lb}$ weight loss over a one year period. The patient had undergone evaluation for these symptoms at her local hospital. A CT scan of the abdomen suggested a pancreatic mass prompting transfer to our institution. On examination, she had notable speech and cognitive delays with scleral telangiectasias, muscle wasting and other features of malnutrition. Her abdominal examination revealed a soft, nondistended abdomen with no palpable masses. Her laboratory studies were remarkable for hypokalemic, hypochloremic metabolic alkalosis, a pre-albumin level of $15 \mathrm{mg} /$ dL (normal: $23-48 \mathrm{mg} / \mathrm{dL}$ ), an IgG level of $461 \mathrm{mg} / \mathrm{dL}$ (normal: $546-1842 \mathrm{mg} / \mathrm{dL}$ ) and an absolute lymphocyte count of 187/cu mm (normal: 1000-4800/cu mm). Other immunoglobulin levels [IgA (186 mg/dL), IgM (70 $\mathrm{mg} / \mathrm{dL})$ and $\operatorname{IgE}(<1 \mathrm{U} / \mathrm{mL}$ ) were within normal limits. She was started on total parenteral nutrition (TPN) immediately upon admission. A repeat CT scan of the abdomen revealed a dilated, hypertrophied stomach consistent with chronic gastric outlet obstruction, abnormal thickening of the antrum and pylorus, and a normal appearing pancreas (Figure 1). Esophagogastroduodenoscopy (EGD) demonstrated diffuse gastritis and esophagitis with a normal appearing duodenum. She was subsequently placed on Pantoprazole ${ }^{\circledast}$, Azithromycin ${ }^{\varpi}$ and Metronidazole ${ }^{\varpi}$ for presumed Helicobacter pylori infection, and kept on continu-

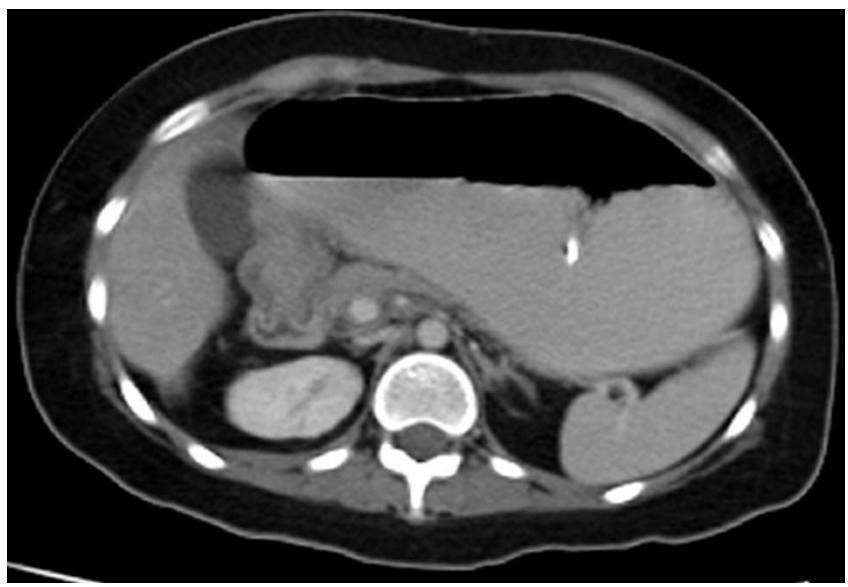

Figure I

CT scan of the abdomen with oral contrast demonstrating markedly dilated stomach, abnormal thickening in the region of the antrum and pylorus of the stomach, and adjacent pancreas thinned due to compression from dilated stomach. There was no intraabdominal adenopathy.

ous nasogastric decompression for persistent emesis. Gastric biopsies identified non-candida fungi on the gastric epithelium and rare lymphoid aggregates in the lamina propria. A Diff-Quik stain for Helicobacter pylori on the biopsied specimen was negative prompting discontinuation of the triple antibody therapy. Several attempts to remove the nasogastric tube were unsuccessful, and so an upper GI series was obtained. This revealed a complete gastric obstruction (Figure 2). To address a possible submucosal infiltrative process such as lymphoma, a repeat

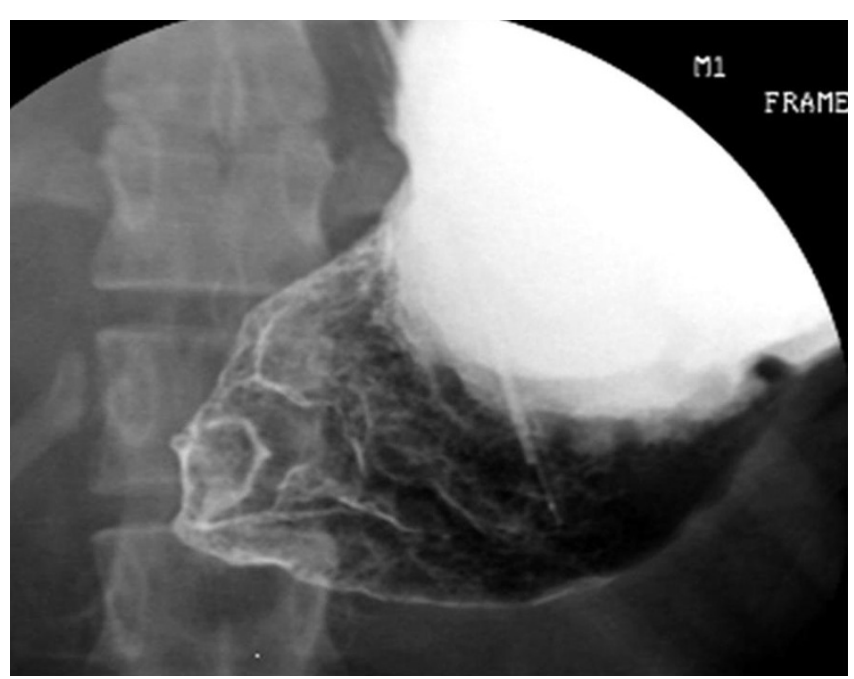

Figure 2

UGI demonstrating complete gastric outlet obstruction. 
EGD was performed to obtain deeper biopsy specimens. At this time, the scope could not be advanced into the duodenum. Deep antral biopsies identified gastric adenocarcinoma. A metastatic workup including chest CT scan and bone scan revealed no evidence of disease. After having received almost 2 weeks of TPN, she was taken to the operating room for exploratory laparotomy. Intra-operatively, she was found to have a large gastric mass involving the distal stomach which was determined to be resectable. During the dissection, there were dense adhesions between the posterior wall of the duodenum and the pancreas that were able to be divided. The tumor was resected by removing $2 / 3$ of the distal stomach, with a minimum of $4.5 \mathrm{~cm}$ margins proximally and $2 \mathrm{~cm}$ margins distally. These margins were free of tumor on frozen and final pathologic exam. The pancreatic surface in the area of resection was biopsied in the operating room due to the finding of adhesions between the stomach and pancreas. The frozen sections showed no evidence of malignancy however, the permanent sections later revealed the presence of a focus of tumor. The patient was reconstructed with a Billroth II gastrojejunostomy after oversewing of the duodenal stump, and a feeding jejunostomy tube and Blake drain were placed. Final histologic evaluation revealed a well to moderately differentiated invasive intestinal-type adenocarcinoma of the stomach with invasion through the muscularis to the serosa, and multifocal vascular and lymphatic invasion.

Postoperatively, the patient initially did well, but then developed increased output from her abdominal drain containing high amylase and lipase levels. An UGI series revealed no evidence of anastamotic leak. On post operative day 7 she developed bloody emesis which resolved spontaneously. However, she developed increasing abdominal pain and a CT scan of the abdomen showed ascites with small bowel obstruction. She was therefore returned to the operating room for exploratory laparotomy which revealed diffuse ascites, an intact gastrojejunostomy, and an obstruction in the afferent limb of the gastrojejunostomy due to large blood clots. The clots were removed via an enterotomy in the afferent limb, and the pancreatic ascites was widely drained. She had a prolonged hospital course but recovered from surgery and was discharged to home on hospital day 48. Two months after surgery she was on continuous jejunostomy tube feeds and eating small amounts by mouth. However, three months after surgery she was refusing to eat, appeared uncomfortable although without complaints, and subsequently declined clinically thereafter. She expired 100 days from initial diagnosis while under hospice care, presumably due to wasting secondary to metastatic adenocarcinoma.

\section{Discussion}

Our patient was the last of three children born at term to a healthy 23 year old mother. Her birth weight was 2.45 $\mathrm{kg}$; there is no family history of congenital immunodeficiency disorder; her two older siblings are healthy and living. The patient was initially seen at age 18 months due to loss of previous ability to walk, poor head control and tendency to walk and fall to the right. By age 3 years, she had progressively worsening ataxia, choreoathetosis, prominent ocular telangiectasias and an elevated alpha fetoprotein of $205.4 \mathrm{ng} / \mathrm{mL}$ (normal 0-6 ng/mL) consistent with ataxia-telangiectasia. All immunoglobulin levels were within normal limits except IgM and IgE, which were slightly depressed. The patient did not receive routine immunoglobulin administration since her disorder was mostly neurologic.

Ataxia-Telangiectasia, an autosomal recessive disorder, is characterized by early onset progressive cerebellar ataxia, oculocutaneous telangiectasia, immune deficiency, and cancer. The causative gene, A-T mutated (ATM) on chromosome 11q22-23, codes for a $350 \mathrm{kDa}$ Ser/Thr protein kinase that belongs to the phosphoinositide 3-kinase (PI3K)-related protein kinase (PIKK) family. ATM with associated proteins function primarily as phosphorylating agents in controlling genomic stability and regulating lymphocyte maturation. Although rare, A-T is the most common primary immunodeficiency syndrome listed in the Immunodeficiency Cancer Registry (ICR), and approximately one-third of A-T patients develop a malignancy during their lifetime. These patients most commonly develop lymphoreticular neoplasms. ATM has also been implicated in breast, lung, head and neck carcinoma, and is associated with a poor prognosis in adult patients with advanced gastric cancer [11].

Gastric adenocarcinoma accounts for the majority of malignant gastric cancer. It arises from the glandular epithelium of the gastric mucosa. The most widely used Lauren histologic classification system divides gastric adenocarcinoma into two types - intestinal and diffuse [12]. The intestinal type, which is usually well-differentiated, originates from recognizable precancerous conditions such as gastric atrophy or intestinal metaplasia. It has a tendency to form glandular structures and spreads to distant organs hematogenously. The diffuse type is typically poorly differentiated, lacks gland formation and is composed of signet ring cells. Early metastases via lymphatic invasion commonly occur. Our patient had moderately differentiated intestinal type adenocarcinoma (Figure 3).

Six cases of gastric cancer associated with A-T have been reported in the literature in patients ages 14-26 years; of these, four cases were reported in the English literature 

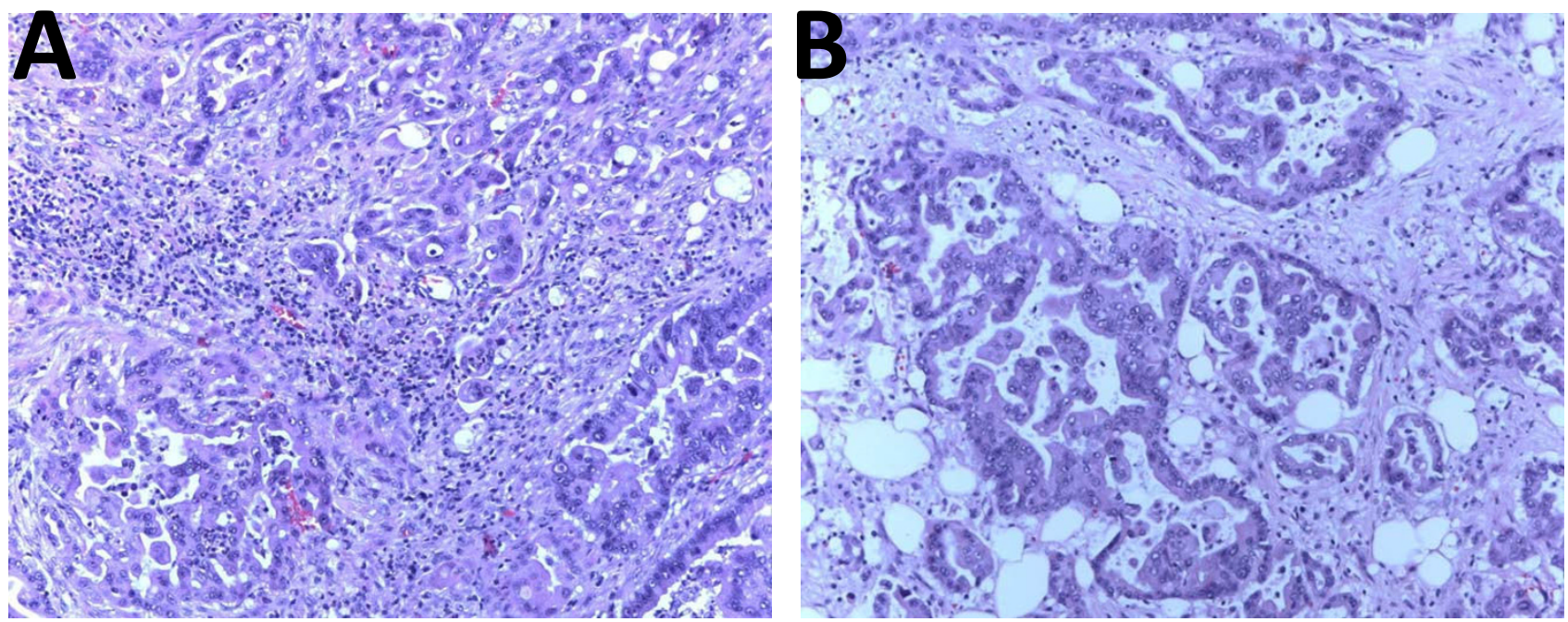

\section{Figure 3}

Hematoxylin and eosin stained sections of the gastric adenocarcinoma resected from our patient. [A] Cytological features of malignant glands; the cells are irregularly shaped with high nucleus to cytoplasm ratio and loss of nuclear polarity. The small dark cells are inflammatory cells (I00X enlargement) [B] This area of tumor is in the serosa; there is redemonstration of irregular glands formed by tumor cells of varying sizes and orientation, with prominent nucleoli. The large clear spaces are fat cells (200x enlargement).

with the last case reported in 1979 (Table 1) [13-15]. A single case series from four different hospitals revealed twelve patients with A-T and associated malignancy, with one patient that probably had gastric mucinous adenocarcinoma [16]. In the normal population, the median age for diagnosis of gastric adenocarcinoma is 70 years. Known risk factors for gastric adenocarcinoma in the general population include Helicobacter pylori infection, atrophic gastritis, a diet high in nitrates and salt, fried or fatty foods, low fruits and vegetables intake, smoking, male gender, and positive family history. As opposed to gastric carcinoma in the general population, gastric carcinoma in A-T patients occurs in the first or second decade of life and has an extremely poor prognosis, with the longest reported survival time of 5 months. It is important to recognize that the risk of gastric carcinoma is also elevated in patients with other primary immune deficiencies such as X-linked agammaglobulinemia, common variable

Table I: Previous cases of gastric adenocarcinoma associated with Ataxia-Telangiectasia Syndrome.

\begin{tabular}{|c|c|c|c|}
\hline Authors & Symptoms on presentation & Age at diagnosis (years)/Sex & Time to death (days) \\
\hline \multirow[t]{2}{*}{$\begin{array}{l}\text { Haerer A et al. (1969) } \\
\text { [13] }\end{array}$} & $\begin{array}{l}\text { Nausea, intractable vomiting and weight } \\
\text { loss }\end{array}$ & $21 / F$ & $?$ \\
\hline & $\begin{array}{l}\text { Abdominal pain, nausea, vomiting and } \\
\text { weight loss }\end{array}$ & $19 / F$ & 90 \\
\hline \multirow{2}{*}{$\begin{array}{l}\text { Kondo } \mathrm{K} \text { and Horikawa } \mathrm{Y}(1975) \\
{[1 \mathrm{I}]}\end{array}$} & Severe mental retardation, gait and speech & $21 / F$ & $?$ \\
\hline & & $22 / \mathrm{F}$ & Gastric cancer confirmed at autopsy \\
\hline $\begin{array}{l}\text { Watanabe A et al. (1977) } \\
\text { [15] }\end{array}$ & $\begin{array}{l}\text { Abdominal pain, vomiting, postive fecal } \\
\text { occult blood test }\end{array}$ & $14 / M$ & 150 \\
\hline $\begin{array}{c}\text { Frais MA (1979) } \\
{[10]}\end{array}$ & Weight loss, anorexia and dyspepsia & $26 / M$ & 5 \\
\hline
\end{tabular}

Year of publication in parentheses 
immune deficiency, and X-HIGM syndromes. In patients with X-linked agammaglobulinemia, chronic atrophic gastritis, intestinal metaplasia and pernicious anemia appear to play an important role in the pathogenesis of gastric adenocarcinoma $[17,18]$. Thus, it is advisable to extend increased awareness of the possibility of gastric carcinoma to the entire population of primary immunodeficient patients.

The A-T patients with gastric carcinoma reported to date presented with non-specific signs of abdominal pain, nausea, vomiting and weight loss. Three patients including ours had initial clinical findings suggestive of ulcer disease. On endoscopy, our patient was noted to have diffuse gastritis, which may have contributed to the development of her adenocarcinoma. All cases reported in the literature had metastatic disease at the time of exploratory laparotomy. Although depressed immunoglobulin levels are associated with increased risk of malignancy, there was no significant correlation between abnormal immunoglobulin levels and the development of gastric cancer in those patients in whom levels were checked, suggesting that injury to the gastrointestinal mucosa in the form of chronic gastritis may predispose these patients to the formation of gastric adenocarcinoma in light of their poor immunologic defense systems. Our patient further demonstrates the challenges associated with diagnosing and caring for these complex patients.

\section{Conclusion}

Although rare, one should consider adenocarcinoma in any patient with ataxia-telangiectasia greater than 10 years of age who presents with non-specific gastrointestinal complaints, since this can lead to earlier diagnosis. Given the poor survival outcome, palliative rather than curative measures may be necessary for unresectable disease. The role of chemo-radiation therapy in A-T patients is limited as it may further predispose the patient to the risk of developing new malignancies [19]. Continuous research efforts will increase our understanding of this disease process and the role of the ATM gene in carcinogenesis.

\section{Consent}

Written informed consent was obtained from the legal guardian for publication of this case report and accompanying images. A copy of the written consent is available for review by the Editor-in-Chief of this journal.

\section{Competing interests}

The authors declare that they have no competing interests.

\section{Authors' contributions}

IAO wrote the manuscript and performed the literature and case review; SFA was involved in the initial operation, performed a detailed literature review, and critically edited the manuscript; SHE performed the upper endoscopies, literature review, and critical review of the manuscript; SH was responsible for all aspects of the manuscript related to the histologic studies; GEB was involved in the patient's initial operation, and assisted in the writing and critical review of the manuscript. All authors read and approved the final manuscript.

\section{References}

I. Syllaba L, Henner K: Contribution a l'independence de l'athetose double idiopathique et congenitale. Rev Neurol (Paris) 1926, I:541-562.

2. Boder E, Sedgwick RP: Ataxia-Telangiectasia: A familial syndrome of progressive cerebellar ataxia, oculocutaneous telangiectasia and frequent pulmonary infection. Pediatrics 1958 , I 2(4):526-554.

3. Woods CG, Taylor AM: Ataxia telangiectasia in the British Isles: the clinical and laboratory features of $\mathbf{7 0}$ affected individuals. QJ Med 1992, 82(298): 169-179.

4. Perlman S, Becker-Catania S, Gatti RA: Ataxia-telangiectasia: diagnosis and treatment. Semin Pediatr Neurol 2003, I 0: 173-I82.

5. Waldmann TA, Mclntire KR: Serum-alpha-fetoprotein levels in patients with ataxia-telangiectasia. Lancet 1972, 2: I I I2-I I I5.

6. Jason JM, Gelfand EW: Diagnostic considerations in ataxia-telangiectasia. Arch Dis Child 1 979, 54:682-686.

7. Stray-Pedersen A, Borresen-Dale AL, Paus E, Lindman CR, Burgers T, Abrahamsen TG: Alpha fetoprotein is increasing with age in ataxia-telangiectasia. Eur J Pediatr Neurol 2007, I I:375-380.

8. Morrell D, Cromartie E, Swift M: Mortality and cancer incidence in 263 patients with ataxia-telangiectasia. J Natl Cancer Inst 1986, 77:89-92.

9. Filipovich $\mathrm{AH}$, Heinitz KJ, Robison LL, Frizzera G: The immunodeficiency cancer registry. Am J Hematol Oncol 1987, 9:183-184.

10. Frais MA: Gastric adenocarcinoma due to ataxia-telangiectasia (Louis-Bar syndrome). J Medical Genetics 1979, 16:160-161.

II. Kang B, Guo RF, Tan XH, Zhao M, Tang ZB, Lu YY: Expression status of ataxia-telangiectasia-mutated gene correlated with prognosis in advanced gastric cancer. Mutation Research 2008, 638:17-25.

12. Lauren P: The two histological main types of gastric carcinoma: diffuse and so-called intestinal-type carcinoma. An attempt at histo-clinical classification. Acta Pathol Microbiol Scand 1965, 64:31-49.

13. Haerer AF, Jackson JF, Evers CG: Ataxia-telangiectasia with gastric adenocarcinoma. JAMA 1969, 210:1884-1887.

14. Kondo K, Horikawa Y: Spinocerebellar degeneration. Ataxiatelangiectasia and malignant tumour. [Japanese]. Naika no Ryôiki (Domain of Internal Medicine) 1975, 35:623-626.

15. Watanabe A, Hanazono $\mathrm{H}$, Sogawa $\mathrm{H}$, Takaya $\mathrm{H}$ : Stomach cancer of a I4-year-old boy with ataxia-telangiectasia. Tohoku J Exp Med 1977, | 2 |: |27-|3|.

16. Murphy RC, Berdon WE, Ruzal-Shapiro C, Hall EJ, Kornecki A, Daneman A, Brunelle $F$, Campbell JB: Malignancies in pediatric patients with ataxia telangiectasia. Pediatr Radiol 1999, 29:225-230.

17. Lavilla P, Gil A, Rodríguez MC, Dupla ML, Pintado V, Fontán G: $\mathbf{X}$ linked agammaglobulinemia and gastric adenocarcinoma. Cancer 1993, 72:1528-1531.

18. Bachmeyer C, Monge M, Cazier A, Le Deist F, de Saint Basile G, Durandy A, Fischer A, Mougeot-Martin M: Gastric adenocarcinoma in a patient with $X$-linked agammaglobulinemia. Eur J Gastroenterol Hepatol 2000, I 2:1033-1035.

19. Makis A, Polychronopoulou S, Haidas S: Osteosarcoma as a second tumor after treatment for primary non-hodgkin's lymphoma in a child with ataxia-telangiectasia. Presentation of a case and review of possible pathogenetic mechanisms. J Pediatr Hematol Oncol 2004, 26:444-446. 